BNL-112482-2016-JA

\title{
Solving local structure around dopants in metal nanoparticles with ab initio modeling of X-ray absorption near edge structure
}

Janis Timoshenko, Atal Shivhare, Robert W. J. Scott, Deyu Lu and Anatoly I. Frenkel

Submitted to Physical Chemistry Chemical Physics

June, 2016

Center for Functional Nanomaterials

Brookhaven National Laboratory

\author{
U.S. Department of Energy \\ DOE Office of Science, \\ DOE Basic Energy Sciences
}

Notice: This manuscript has been authored by employees of Brookhaven Science Associates, LLC under Contract No. DE- SC0012704 with the U.S. Department of Energy. The publisher by accepting the manuscript for publication acknowledges that the United States Government retains a non-exclusive, paid-up, irrevocable, world-wide license to publish or reproduce the published form of this manuscript, or allow others to do so, for United States Government purposes. 


\section{DISCLAIMER}

This report was prepared as an account of work sponsored by an agency of the United States Government. Neither the United States Government nor any agency thereof, nor any of their employees, nor any of their contractors, subcontractors, or their employees, makes any warranty, express or implied, or assumes any legal liability or responsibility for the accuracy, completeness, or any third party's use or the results of such use of any information, apparatus, product, or process disclosed, or represents that its use would not infringe privately owned rights. Reference herein to any specific commercial product, process, or service by trade name, trademark, manufacturer, or otherwise, does not necessarily constitute or imply its endorsement, recommendation, or favoring by the United States Government or any agency thereof or its contractors or subcontractors. The views and opinions of authors expressed herein do not necessarily state or reflect those of the United States Government or any agency thereof. 
BNL-112482-2016-JA

Cite this: DOI: $10.1039 / \mathbf{x x x x x x x x x x}$

Received Date

Accepted Date

DOI: $10.1039 / x x x x x x x x x x$

www.rsc.org/journalname

\section{Solving local structure around dopants in metal nanoparticles with ab initio modeling of X-ray absorp- tion near edge structure}

\author{
Janis Timoshenko, ${ }^{* a}$ Atal Shivhare, ${ }^{b}$ Robert W. J. Scott, ${ }^{b}$ Deyu Lu, ${ }^{c}$ and Anatoly I. \\ Frenkel $^{* a}$
}

\begin{abstract}
We adopted ab-initio X-ray Absorption Near Edge Structure (XANES) modelling for structural refinement of local environments around metal impurities in a large variety of materials. Our method enables both direct modelling, where the candidate structures are known, and the inverse modelling, where the unknown structural motifs are deciphered from the experimental spectra. We present also estimates of systematic errors, and their influence on the stability and accuracy of the obtained results. We illustrate our approach by following the evolution of local environment of palladium atoms in palladium-doped gold thiolate clusters upon chemical and thermal treatments.
\end{abstract}

\section{Introduction}

During the last decades X-ray absorption spectroscopy (XAS) appeared to be an invaluable tool for structural studies ${ }^{1-3}$, with many applications in chemistry ${ }^{4,5}$. XAS is a chemically sensitive method and allows one to probe the local environment around selected type of atoms. The particular advantage of XAS method is its applicability to a broad range of materials, including crystalline, disordered, liquid and nanostructured materials, and it allows one to investigate concentrated as well as very diluted samples ${ }^{2,3}$. Another important advantage of XAS approach is its applicability to time-resolved studies ${ }^{6}$ due to the short timescale $\left(10^{-15}-10^{-16} \mathrm{~s}\right)$ of the photoabsorption process ${ }^{3}$, and also its applicability to in-situ and operando studies ${ }^{4}$ due to highpenetration depth of X-rays that allows one to investigate samples in complex environments, such as reaction chambers 5,7 , and in extreme conditions ${ }^{8-10}$. Therefore XAS is a premier method for studying catalytic mechanisms, because of its excellent ability to probe the evolution of structure and electronic state of catalysts in the course of chemical reactions.

While extended X-ray absorption fine structure (EXAFS) is commonly used for solving the unknown local structure around $\mathrm{X}$ ray absorbing species, its quantitative power is severely limited by heterogeneity of the sample, where the absorbing species are present in different chemical environments. In the cases typical

\footnotetext{
${ }^{a}$ Physics Department, Yeshiva University, New York, NY 10016, USA. Tel: +1 631344 3494; E-mails: janis.timoshenko@gmail.com,anatoly.frenkel@yu.edu

${ }^{b}$ Department of Chemistry, University of Saskatchewan, 110 Science Place, Saskatoon, SK S7N 5C9, Canada

${ }^{c}$ Center for Functional Nanomaterials, Brookhaven National Laboratory, Upton, NY 11973, USA
}

for chemical transformations where X-ray resonant atoms often coexist in reduced and oxidized states and/or in multiple crystalline forms, the acquired EXAFS data correspond to an ensemble average of contributions of these species, and their separate quantitative analyses are extremely difficult ${ }^{11}$.

Another typical limitation of EXAFS analysis in the case of nanoscale systems are large effects of the bond length disorder and reduction in coordination numbers that reduce amplitudes of EXAFS oscillations and lower the signal-to-noise ratio. These factors decrease the structural sensitivity of EXAFS technique to more distant coordination shells and thus limit its ability to characterize the three-dimensional geometry of the atomic arrangement in nanoscale systems, when their dimensions are in the range of ca. $2 \mathrm{~nm}$ or less. Finally, another limitation of EXAFS in the case of nanomaterials is the strongly asymmetric bond length disorder, which is typical for metal particles of that size ${ }^{12-16}$. Asymmetric bond length distributions are responsible for the known artifacts in EXAFS analysis and result in underestimation of coordination numbers and bond length variances, further lowering the efficiency of EXAFS for structural and dynamic characterization of nanocatalysts ${ }^{14-16}$.

Another part of XAS, X-ray absorption near edge structure (XANES) spectroscopy, is an attractive complement or alternative to EXAFS analysis, and mostly does not suffer from the deficiencies outlined above. XANES is less sensitive to disorder effects, and XANES data of a good quality can be acquired at harsher reaction conditions and with better time resolution than most EXAFS data ${ }^{17}$. While XANES part of X-ray absorption spectrum is most sensitive to the local electronic structure, determined mostly by the valence state of the absorbing atom and the local point group symmetry of the absorbing site ${ }^{18}$, recent findings (both 
experimental and theoretical) suggest that more complex information on the geometrical arrangement of atoms around the absorbing atom can be recovered from the analysis of XANES features ${ }^{17,19-23}$ and, hence, can provide possibilities for a robust, XANES-based refinement of materials structure.

While XANES modeling has not yet achieved the same quantitative capability in structural refinement as is in the case of EXAFS analysis, the progress in XANES theory during the last decades was remarkable ${ }^{18,22,24}$. Direct fitting of XANES spectra to obtain values of structure parameters currently is provided by such codes as MXAN ${ }^{25,26}$ and FITIT ${ }^{27,28}$, which are able to provide promising results for simple systems, such as small organometallic molecules. The applicability of these approaches is, nevertheless, currently limited to well-defined, homogeneous materials with a few degrees of freedom. Similarly as EXAFS data fitting, these XANES fitting approaches are not useful for the analysis of heterogeneous systems.

On the other hand, for quantitative XANES studies of heterogeneous mixtures there is a number of well-known linear algebrabased tools available. Most widely used approaches are Linear Combination Analysis (LCA) ${ }^{29}$, Principal Component Analysis (PCA) ${ }^{30-33}$ and Multivariate Curve Resolution with Alternating Least Squares (MCR-ALS) fitting ${ }^{34-37 .}$

LCA method requires the knowledge of the XANES spectra of "standard" materials, because such spectra are linearly combined in this method to represent the data of the unknown material. Knowledge of XANES spectra of reference materials is required also for PCA and MCR-ALS analysis to correlate the signal components, yielded by these methods, with physical structure models. For many cases synthesis of such reference samples can be challenging, or even impossible. For instance, experimental XANES spectrum measured from the dopants in a metal nanoparticle is a mixture of spectra, each corresponding to a dopant located at a particular site in the nanoparticle (e.g., on the surface or in the core). Synthesizing the corresponding experimental standards for each dopant location is an almost impossible, and surely not practical task, hence, a conventional LCA or PCA analysis aimed at their speciation is not a feasible approach.

Our alternative approach is based on the replacement of the experimental XANES standards by the results of ab-initio XANES simulations. In order to replace experimental standards by theoretical ones, we should investigate first the systematic errors in theoretical calculations and their effects on the structural refinement. We use simple models of binary and ternary mixtures of experimental spectra in model compounds and their theoretical XANES spectra to solve the speciation problem, and compare the results with the known mixing fractions of species. We demonstrate that LCA fit employing theoretically calculated standards provides a reasonable accuracy for the determination of weights of different contributions, as expected.

As the next step, we complement the information from ab-initio XANES modeling with the information available from experimental data to reduce the influence of systematic error and to improve the accuracy of the analysis. For this purpose we tested an alternative approach to XANES analysis, based on the blind signal separation (BSS) ${ }^{38,39}$, guided by the information from ab-initio
XANES simulations. Using this combined procedure, we demonstrate that even without experimental XANES data for reference materials one can estimate the ratio of different components in multiphase material or the ratio of different non-equivalent sites with accuracy in their mixing fractions better than $10 \%$.

Finally, we apply this method to analyze the placement of Pd dopants in gold thiolate clusters and to investigate the structural evolution of these systems upon chemical and thermal treatments. For this purpose we chose the monodispersed $\mathrm{Au}_{25}\left(\mathrm{SC}_{8} \mathrm{H}_{9}\right)_{18}$ clusters doped with $\mathrm{Pd}$, a potentially promising catalyst due to the possibility to tailor its electronic properties by changing the type of dopant atoms and their location within the cluster $^{40-43}$.

\section{Samples and experimental data}

Experimental data for this work were acquired previously and the details on synthesis and experiments are available in the literature $^{44}$. We will briefly describe the synthesis and treatment of the gold thiolate clusters because of their special role as an illustration of our structural refinement procedure.

Synthesis of highly monodispersed $\mathrm{Au}_{25}\left(\mathrm{SC}_{8} \mathrm{H}_{9}\right)_{18}$ clusters, where one of the gold atoms is replaced by Pd atom, is described in Refs. ${ }^{44,45}$. Here we just emphasize that the process has been carried out in two steps. First, $\mathrm{Au}_{25}\left(\mathrm{SC}_{8} \mathrm{H}_{9}\right)_{18}$ clusters without $\mathrm{Pd}$ were synthesized. Secondly, solution of thiol-protected gold clusters was reacted with 0.5 equiv or 6.0 equiv of $\mathrm{Pd}$ acetate, and stirred overnight. Formation of $\mathrm{Pd}_{x} \mathrm{Au}_{25}$ clusters was evidenced by UV-vis spectroscopy and TEM measurements (see Ref. ${ }^{44}$ for details). Note that this approach to synthesis of bimetallic clusters differs from the more conventional co-reduction method $42,46,47$.

For catalytic applications activation of the synthesized clusters can be accomplished by removing the thiols from clusters surface via chemical or thermal treatments. Therefore, in addition to the analysis of as-prepared samples, in this study we use Pd K-edge XANES analysis to investigate changes in Pd-doped $\mathrm{Au}_{25}$ clusters upon two different treatments. In the first case (thermal treatment), samples were calcinated at $250^{\circ} \mathrm{C}$ for 90 minutes in air, and then treated for 90 minutes in $\mathrm{H}_{2}$ atmosphere. In the second case (chemical treatment), $0.1 \mathrm{~mL}$ of $\mathrm{LiBH}_{4}$ were added to the $100 \mathrm{mg}$ of as-prepared sample, suspended in hexane, and then sample was washed and dried.

In addition, in this study to test the proposed method for XANES data analysis, we also used Pd K-edge XANES for three bulk compounds: Pd foil, palladium oxide PdO in powder form and palladium sulphide PdS in powder form. XAS measurements for these standard compounds were also carried out in transmission mode.

Processing of raw synchrotron XAS data (aligning, merging, background subtraction and edge-step normalization) was carried out using Athena software ${ }^{48}$.

\section{Ab-initio XANES calculations}

In this study all Pd K-edge XANES calculations were performed with FEFF (version 9.6.4) ${ }^{49}$ self-consistent code within full multiple scattering (FMS) approach and muffin-tin (MT) approximation. The calculation parameters were chosen to reproduce as 
well as possible the Pd K-edge XANES for Pd foil (face-centered cubic (fcc) structure). For all cases we perform XANES simulations for material equilibrium structure, known from diffraction experiments, i.e., the influence of thermal disorder is not accounted for in our calculations.

We have found out that a reasonable agreement between experiment and theory can be obtained with complex exchangecorrelation Hedin-Lundqvist potential and with conventional fully-screened core-hole model. Default values of MT radii were used, as provided within FEFF code. We also have found out that for bulk materials the obtained results are not very sensitive to the value of chosen FMS cluster size $R_{\mathrm{FMS}}$, and the main features are reproduced with $R_{\mathrm{FMS}}=5.0 \AA$. No significant changes in the shape of spectra are observed for $R_{\mathrm{FMS}}>7.0 \AA$, and therefore we used this value for all further calculations for bulk materials. For nanoclusters, $R_{\mathrm{FMS}}$ was chosen at a large enough value so that the whole cluster is included in the FMS calculations. No broadening, except due to the core-hole, was included in XANES calculations. The calculated absorption spectra $\tilde{\mu}(E)$ were shifted in energy by $\Delta E$ to align the energy scale used in FEFF calculations with the energy scale of experimental data. The value of $\Delta E=23.8 \mathrm{eV}$ was chosen as the one that gave the best agreement between experimental and calculated Pd K-edge XANES for Pd foil, and was fixed for all further calculations. After the alignment, the theoretical spectra spanned energies from $E_{\min }=24347 \mathrm{eV}$ to $E_{\max }=24429$ $\mathrm{eV}$. Spectra were calculated on a non-uniform grid with step size of $0.1 \mathrm{eV}$ for data points near absorption edge. Step size increased linearly with energy, and reached $1.7 \mathrm{eV}$ at $E=E_{\max }$. The presence of hydrogen atoms in the $\mathrm{Au}_{25}$ structure was ignored in our calculations.

\section{Quantitative analysis of XANES data}

\subsection{Linear component analysis for model data}

We start with calculations for common Pd-containing bulk materials with known structures. We have performed ab-initio XANES modeling for (i) palladium metal with fcc structure, (ii) palladium oxide PdO with tetragonal structure ${ }^{50}$ and (iii) palladium sulphide PdS with tetragonal structure ${ }^{51}$.

Comparison of experimental and calculated spectra and also comparison of their first derivatives, which can highlight the subtle features near the absorption edge, are shown in Fig. 1. Note that the overall agreement between theory and simulations is good for all three considered materials. In the extended energy range (in this case with $E$ larger than ca. $24390 \mathrm{eV}$ ) the influence of disorder effects starts to play an important role.

To estimate the accuracy of the LCA approach, we construct several model spectra as linear combinations of experimental data for the reference compounds, described above:

$$
\mu(E)=w_{\mathrm{Pd}} \mu_{\mathrm{Pd}}(E)+w_{\mathrm{PdO}} \mu_{\mathrm{PdO}}(E)+w_{\mathrm{PdS}} \mu_{\mathrm{PdS}}(E),
$$

where $\mu_{i}(E)$ are normalized experimental XANES spectra for Pd foil, PdO and PdS, and $w_{i}$ are the corresponding weights of given contribution to the total spectrum. We require that $0 \leq w_{i} \leq 1$ and $\sum_{i} w_{i}=1$. Some of the constructed model spectra are shown in Fig.2.

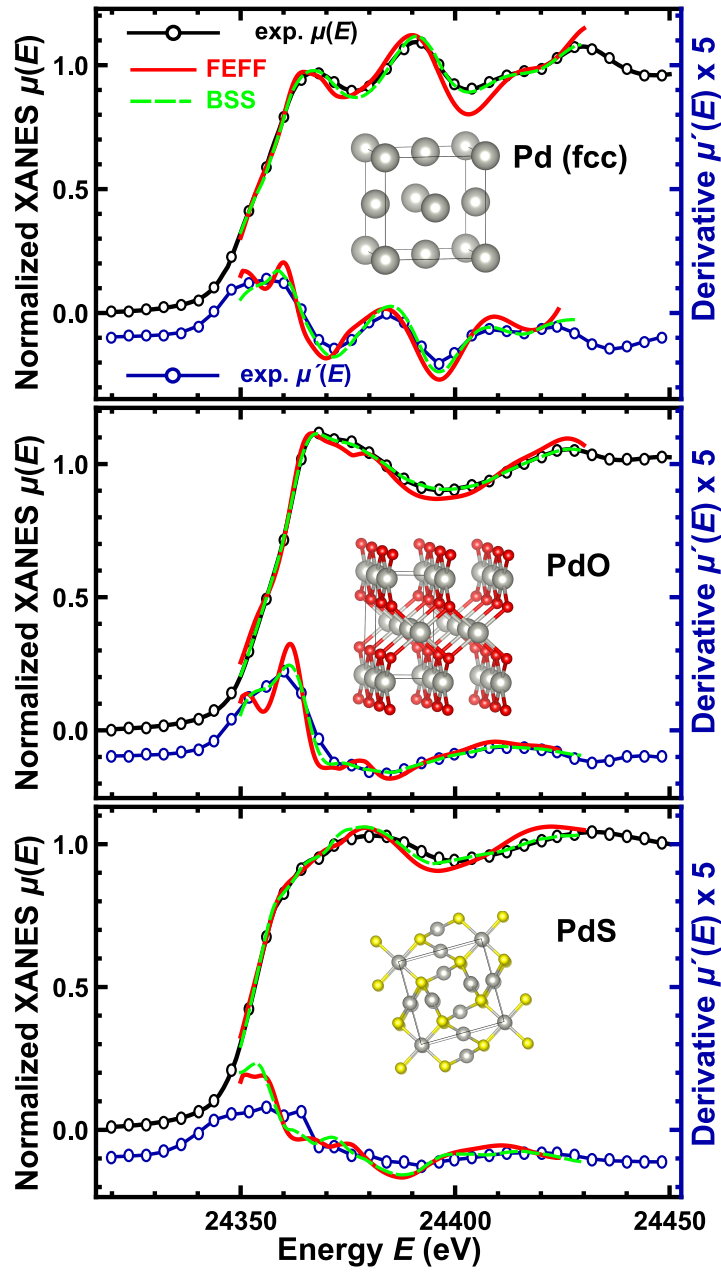

Fig. 1 (Color online) Pd K-edge XANES spectra for bulk reference materials: palladium foil, $\mathrm{PdO}$ and PdS. Experimental data (normalized XANES spectra and their derivatives) are compared with the results of FEFF calculations. Green dashed lines show the corresponding theoretical spectra, obtained using BSS-like procedure, as discussed in Section 4.2.

Next, we fit these model data using linear combinations of theoretical signals:

$$
\tilde{\mu}(E)=\tilde{w}_{\mathrm{Pd}} \tilde{\mu}_{\mathrm{Pd}}(E)+\tilde{w}_{\mathrm{PdO}} \tilde{\mu}_{\mathrm{PdO}}(E)+\tilde{w}_{\mathrm{PdS}} \tilde{\mu}_{\mathrm{PdS}}(E),
$$

where $\tilde{\mu}_{i}(E)$ are calculated XANES spectra for Pd foil, PdO and PdS, and coefficients $\tilde{w}_{i}$ are obtained using least-square fitting. Best-fitted curves $\tilde{\mu}(E)$ are shown as red dashed lines in Fig.2. We can then characterize the error of the reconstruction by quantity $\Delta$ defined as:

$$
\Delta=\sqrt{\sum_{i}\left(\tilde{w}_{i}-w_{i}\right)^{2}} .
$$

The obtained values of parameter $\Delta$ as a function of the weights $w_{i}$, used to construct the model spectra, are shown in Table 1 and plotted in Fig. 3. In many cases, especially when the mixing ratios of all three components are close, this approach is able to reconstruct the original weights quite accurately. However, when the weight of one of the components is reduced and its contribution 


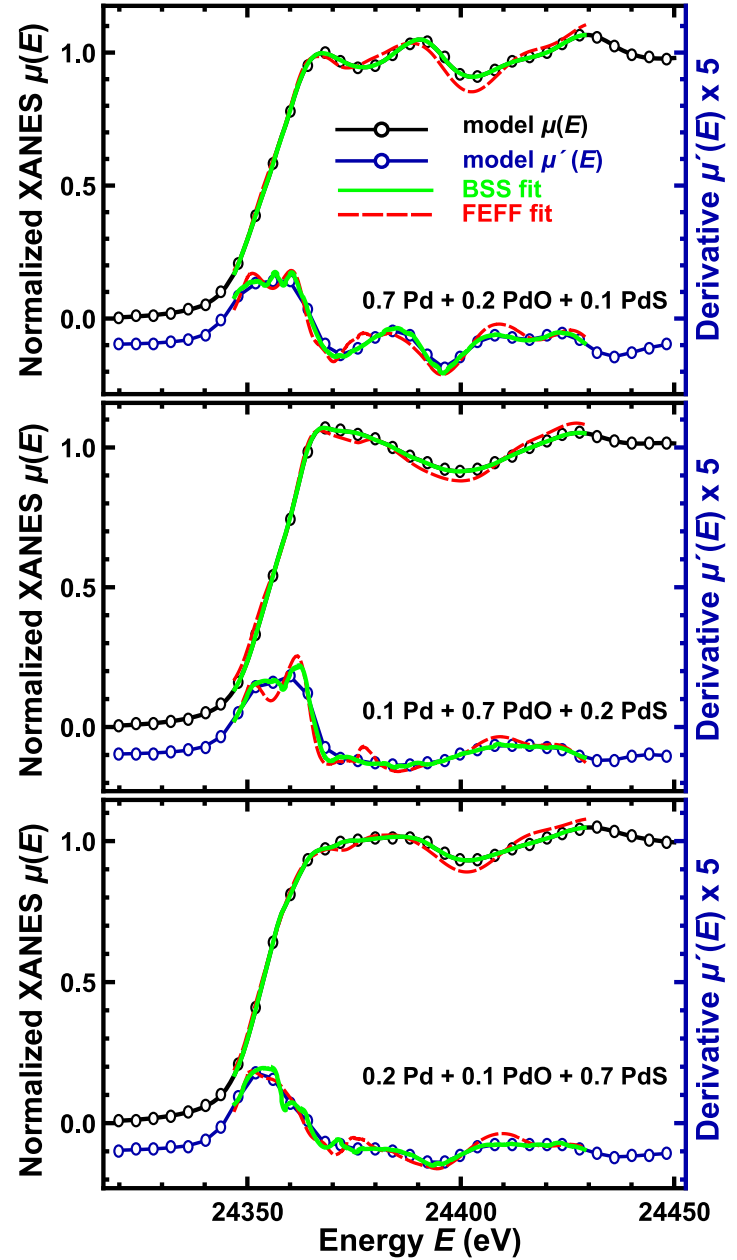

Fig. 2 (Color online) Model XANES spectra, obtained as linear combination of experimental Pd, PdO and PdS XANES data, and their fits using linear combinations of standards that are obtained in FEFF calculations and in BSS-like procedure.

to the total spectrum gets comparable with the statistical error of XANES simulations, estimated $\tilde{w}_{i}$ can differ from the true values of weights $w_{i}$ significantly. The $\Delta$ values are increasing in this case and can reach the values as high as 0.35 , when contribution of metallic Pd to the total spectrum is dominating. The average $\Delta$ value, obtained in this study of model data, is about 0.15 .

\subsection{Simulations-guided blind signal separation}

To improve the accuracy of XANES analysis using theoretically calculated reference spectra, we propose the following scheme, which is applicable when experimental data for several samples with different compositions (different sets of weights $w$ ) are available.

Let us denote the XANES spectra of $n$ reference materials as $s_{j}(E), j=1, \ldots, n$. The available $k$ experimental XANES spectra $\mu_{i}(E), i=1, \ldots, k$ then can be expressed as:

$$
\mu_{i}(E)=\sum_{j=1}^{n} w_{i j} s_{j}(E),
$$

where neither the coefficients $w_{i j}$ nor the reference spectra $s_{j}$ are
Table 1 Results of LCA fits of model spectra: estimations of weights for $\mathrm{Pd}, \mathrm{PdO}$ and $\mathrm{PdS}$ contributions, obtained using (i) standards that are directly calculated by FEFF and (ii) standards that are obtained in BSS-like procedure. True values of corresponding contribution to the total model spectrum are also given for comparison

\begin{tabular}{lllll}
\hline & $w_{\text {Pd }}$ & $w_{\text {PdO }}$ & $w_{\text {PdS }}$ & $\Delta$ \\
\hline True value & 0.70 & 0.20 & 0.10 & - \\
FEFF & 0.55 & 0.18 & 0.27 & 0.23 \\
BSS & 0.61 & 0.19 & 0.20 & 0.14 \\
\hline True value & 0.10 & 0.70 & 0.20 & - \\
FEFF & 0.12 & 0.67 & 0.21 & 0.04 \\
BSS & 0.09 & 0.71 & 0.19 & 0.02 \\
\hline True value & 0.20 & 0.10 & 0.70 & - \\
FEFF & 0.28 & 0.06 & 0.65 & 0.10 \\
BSS & 0.29 & 0.07 & 0.64 & 0.11 \\
\hline True value & 0.33 & 0.33 & 0.34 & - \\
FEFF & 0.31 & 0.30 & 0.38 & 0.05 \\
BSS & 0.33 & 0.32 & 0.35 & 0.01 \\
\hline
\end{tabular}

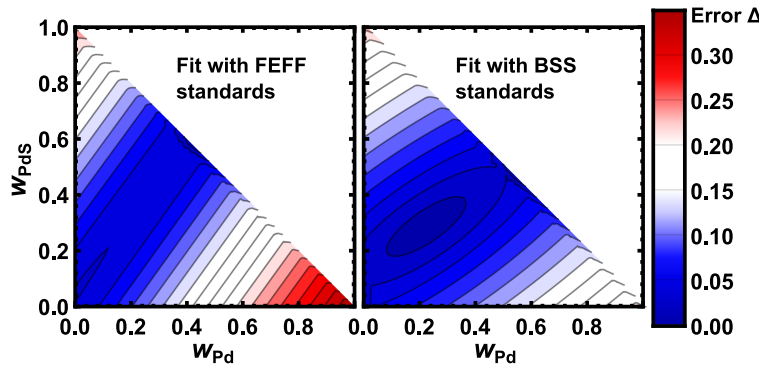

Fig. 3 (Color online) LCA fit errors $\Delta$ as a function of the true values of $\mathrm{Pd}$ and PdS weights in the total model spectrum. Results obtained with standards that are directly calculated by FEFF (left panel) and with standards that are obtained in BSS-like procedure (right panel) are compared.

known. This set of equations can be rewritten in matrix form as $\mathbf{M}=\mathbf{W S}$, where $\mathbf{M}$ is a vector consisting of the measured spectra, $\mathbf{S}$ is a vector consisting of the unknown standard (or reference) spectra and $\mathbf{W}$ is matrix, formed by coefficients $w_{i j}$.

The problem of finding matrices $\mathbf{W}$ and $\mathbf{S}$ is called blind signal separation (BSS) problem ${ }^{38,39,52}$. It is solvable only if some additional constraints are imposed on the elements of $\mathbf{W}$ and S. PCA can be considered one of the examples of the BSS methods, where we constrain $\mathbf{S}$ elements to be mutually orthogonal ${ }^{30,38}$. Another approach, often used for BSS problems, is individual component analysis (ICA), where, in turn, we require $\mathbf{S}$ elements to be statistically independent ${ }^{39}$. Finally, the non-negative matrix factorization $^{38,52}$ (NNMF) requires all elements of matrices $\mathbf{W}$ and $\mathbf{S}$ to be non-negative. Obviously, the problem that we want to solve in XANES analysis is closely related to the problems, treated by NNMF approach. Note also that BSS and NNMF approaches are similar to MCR-ALS method, used for interpretation of spectroscopic data ${ }^{34-36,53}$.

Unfortunately, the non-negativity of matrices $\mathbf{W}$ and $\mathbf{S}$ does not guarantee uniqueness of solution. Therefore some other constraints are required in NNMF approaches ${ }^{52}$. In our case as such an additional constraint we propose to use the information obtained in ab-initio XANES simulations.

We construct a demixing matrix $\mathbf{V}$, so that estimation $\tilde{\mathbf{S}}$ of reference spectra can be obtained as $\tilde{\mathbf{S}}=\mathbf{V M}$. We request that BSS 
estimators of reference spectra $\tilde{s_{i}}$ should be as close as possible to the corresponding spectra $s_{i}^{\mathrm{FEFF}}$, calculated by FEFF code, i.e., the Euclidean distances between $\tilde{s}_{i}$ and $s_{i}^{\mathrm{FEFF}}$ should be as low as possible. Since the matrix $\mathbf{V}$ inverts matrix $\mathbf{W}$, we can construct $\mathrm{V}$ as

$$
\mathbf{V}=\left(\tilde{\mathbf{W}}^{\mathbf{T}} \tilde{\mathbf{W}}\right)^{-\mathbf{1}} \tilde{\mathbf{W}}^{\mathbf{T}},
$$

Here $\tilde{\mathbf{W}}$ is $k \times n$ matrix, consisting of variables $\tilde{w}_{i j}$. We obtain the values of $\tilde{w}_{i j}$ using optimization procedure, which should minimize $\left\|\mathbf{V M}-\mathbf{S}^{\mathrm{FEFF}}\right\|$ (the Euclidean distance between BSS prediction and results of FEFF calculations), requesting additionally that for all $i$ and $j$ corresponding $\tilde{w}_{i j} \geq 0$ and $\sum_{j=1}^{n} w_{i j}=1$. To solve this non-linear optimization problem, we apply NelderMead method ${ }^{54}$. Such direct fitting approach is feasible if the total number of reference spectra is small. The obtained estimations of reference spectra $\tilde{s_{i}}(E)$ then can be used in LCA fitting instead of $s_{i}^{\mathrm{FEFF}}(E)$.

Obviously, the total number of experimental spectra for $\mu_{i}(E)$ for this BSS-like procedure should be at least equal to the number of reference spectra $s_{i}(E)$. Larger sets of experimental spectra can be used to reduce the influence of experimental noise in the data and to stabilize the procedure numerically.

To validate the proposed approach, we applied it to the model data, constructed as linear combinations of experimental Pd Kedge XANES spectra for Pd foil, PdO and PdS bulk materials. As input for our BSS-like procedure, we used 3 model spectra, shown in Fig. 2. As a result, we obtained estimators for Pd, PdO and PdS spectra that are compared with the experimental XANES spectra for these materials in Fig. 1. As one can see from Fig. 1, the results of our BSS-like procedure (green lines) are significantly closer to the true experimental XANES data (black lines and circles) for these reference materials than the direct results of FEFF simulations (red lines).

We used the obtained estimators for reference spectra to perform linear combination fitting of model data, constructed with different weights for Pd, PdO and PdS contributions. Note that the same reference spectra estimators were used for each of the fits. The errors of the analysis, characterized by parameter $\Delta$, are shown in Table 1 and are plotted in the right panel of Fig. 3. As one can see from this Figure, the errors, obtained using BSS estimators, are smaller, i.e., the obtained results are more reliable than the ones, obtained using FEFF data directly (left panel of Fig. 3). Especially pronounced improvement is observed in the cases, when contributions of some of the components in the mixture are comparable with the systematic errors of FEFF simulations. Note that the maximal value of $\Delta$ is reduced from 0.35 to 0.24 in this case.

\section{XANES analysis for palladium-doped gold thiolate clusters}

In this Section we apply the proposed method to the interpretation of Pd K-edge XANES spectra for palladium-doped gold thiolate clusters. Experimental Pd K-edge XAS data for this material are shown in Fig. 4 and 5.

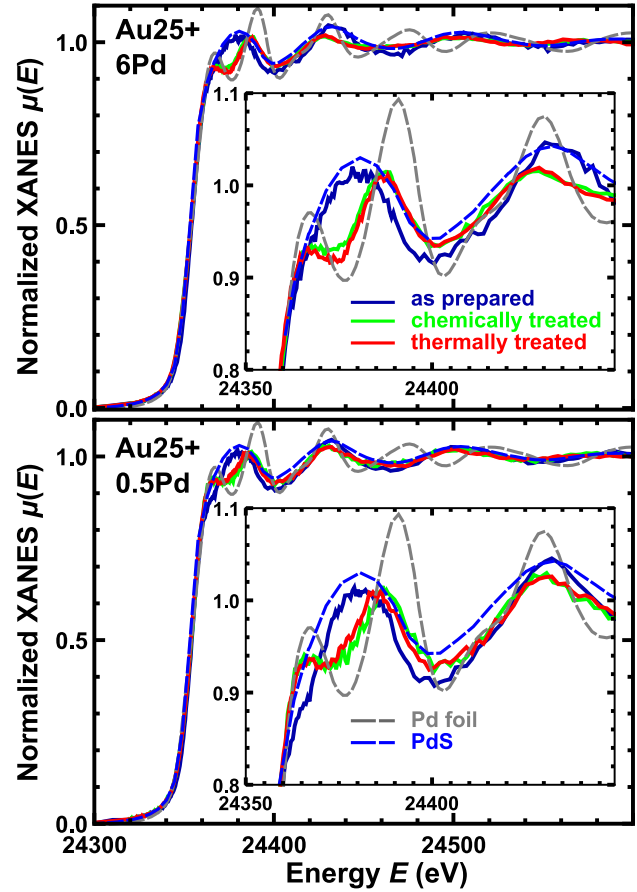

Fig. 4 (Color online) Experimental Pd K-edge XAS spectra for samples, obtained in a reaction of thiolated $\mathrm{Au}_{25}$ clusters and 6.0 equiv of $\mathrm{Pd}$ acetate ("Au25 + $6 \mathrm{Pd}$ ") and 0.5 equiv of Pd acetate ("Au25 + $0.5 \mathrm{Pd}$ "). Experimental Pd K-edge XAS spectra for Pd foil and PdS are also shown for comparison.

Single crystal diffraction data $47,55,56$ and theoretical simulations ${ }^{43}$ suggest that $\mathrm{Au}_{25}$ clusters with and without Pd doping have a structure, schematically shown in the inset in Fig. 5. Pure gold clusters consist of icosahedral $\mathrm{Au}_{13}$ core, to which 6 $-\mathrm{S}-\mathrm{Au}-\mathrm{S}-\mathrm{Au}-\mathrm{S}-$ motifs, so called "staples", are attached ${ }^{44}$. When one of the gold atoms is replaced by Pd, it can be located in one of the three non-equivalent positions: in the core, at the surface or within the staple motif.

Theoretical simulations ${ }^{43}$ show that the cluster with Pd in the core is the most stable configuration. And, indeed, EXAFS studies, Mössbauer spectroscopy and single crystal diffraction studies suggest that for samples, prepared via conventional co-reduction method, Pd is most likely located in the cluster core 47,55 . However, recent EXAFS study of $\mathrm{PdAu}_{25}$ clusters, synthesized via postsynthetic treatment of gold clusters, showed significant contribution of Pd-S bonds, thus implying that Pd in this case is located within the staple motif ${ }^{44}$. Pd K-edge XANES data and Fourier transformed (FT) EXAFS data, shown in Fig. 4 and 5, support this finding: clearly, both EXAFS and XANES parts of X-ray absorption spectra for as-prepared samples exhibit strong similarity with corresponding spectra for palladium sulphide.

Thermal or chemical treatments may be used to partially remove the thiols and activate the material for catalytic applications. Available EXAFS data (Fig. 5) are in agreement with the expected reduction of the total number of $\mathrm{Pd}-\mathrm{S}$ bonds upon chemical and thermal treatment, as evidenced by the decrease in the amplitude of FT peak, corresponding to $R=1.5-2.0 \AA$. The increase of the contribution to the FT with $R=2.5-3.0 \AA$ for 


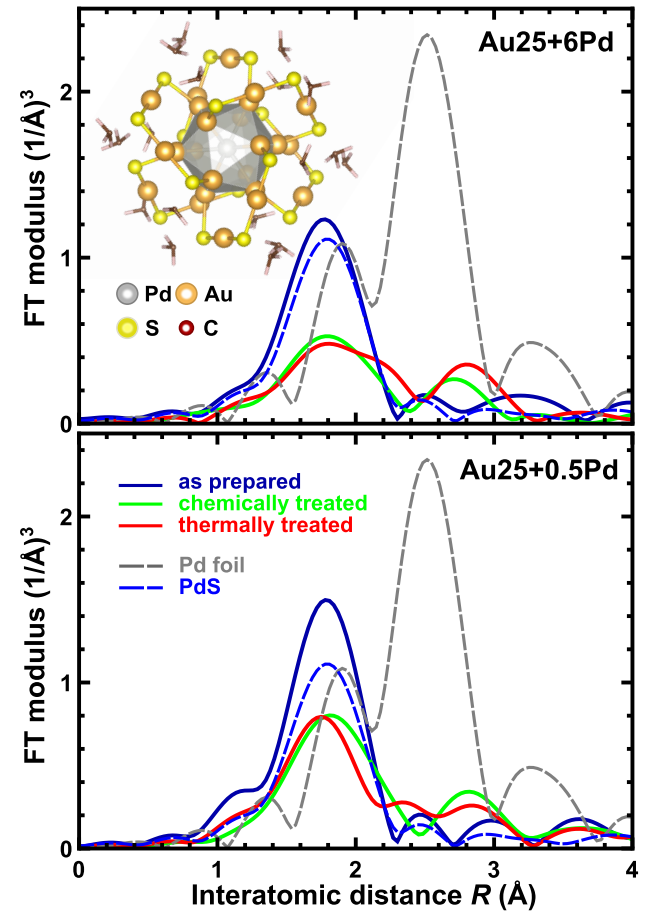

Fig. 5 (Color online) Fourier-transformed (FT) experimental Pd K-edge EXAFS spectra for samples, obtained in a reaction of thiolated $\mathrm{Au}_{25}$ clusters and 6.0 equiv of $\mathrm{Pd}$ acetate ("Au25 $+6 \mathrm{Pd}$ ") and 0.5 equiv of $\mathrm{Pd}$ acetate ("Au25 + $0.5 \mathrm{Pd}$ ") ${ }^{44}$. Experimental Pd K-edge EXAFS spectra for Pd foil and PdS are also shown for comparison. In the inset structure model of thiolated $\mathrm{Au}_{25}$ cluster is shown ${ }^{47}$.

treated samples, in turn, may be interpreted as an evidence of $\mathrm{Pd}-\mathrm{Au}$ bonds formation.

At the same time there are indications that EXAFS analysis cannot be used as a reliable tool to investigate this system. Quantitative EXAFS analysis of as-prepared samples was carried out in Ref. ${ }^{44}$, and it was obtained that coordination number for Pd-S bond is close to 3.6. This result is in a contradiction with the structure model, as shown in the inset of Fig. 5: even if all Pd is located in the staples, the maximal possible coordination number for Pd-S bond is 2 . The high coordination number for Pd-S, as observed by EXAFS, may be an evidence of presence of some other $\mathrm{Pd}-\mathrm{S}$ containing species in the samples and/or, as speculated in Ref. ${ }^{44}$, it may be a result of cross-linking of staples, belonging to the neighboring gold clusters. In any case, this finding indicates that any reduction in $\mathrm{Pd}-\mathrm{S}$ coordination number, observed by EXAFS, cannot be interpreted simply as a reduction in the number of staples, but can also reflect the changes in the ratio of Pd with 2 and more sulphur atoms in the first coordination shell.

Moreover, while the as-prepared clusters are monodisperse, one can expect that upon chemical and thermal treatment they will grow or agglomerate ${ }^{57}$. Hence the measured experimental XAS data may be averaged over many different nanoparticles, which makes quantitative EXAFS analysis challenging ${ }^{11}$. In addition, pronounced non-Gaussian disorder is expected for such small nanoclusters, which may have significant influence on EXAFS spectrum. As demonstrated in Ref. ${ }^{15}$, in such cases the conventional EXAFS analysis can underestimate the coordination

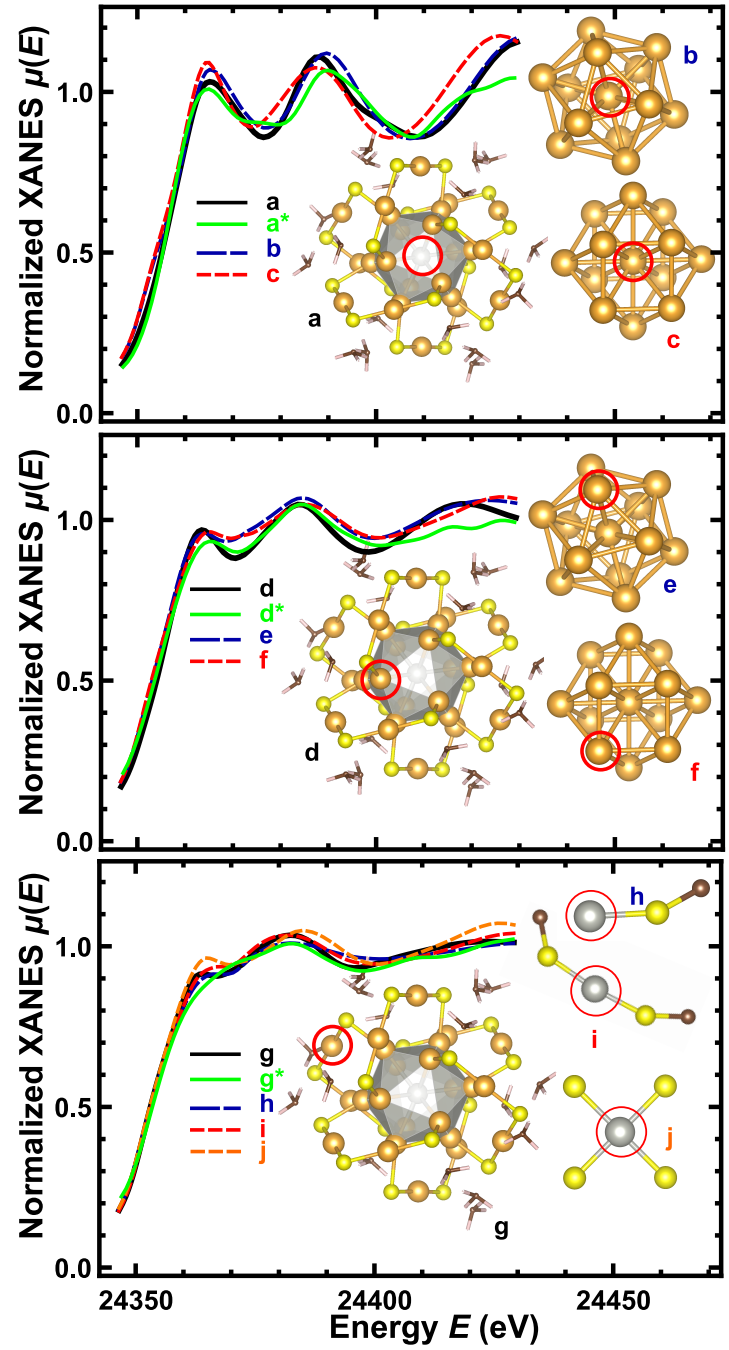

Fig. 6 (Color online) Calculated Pd K-edge XANES spectra for Pd atom, located in the core of $\mathrm{Pd}$-doped thiolated $\mathrm{Au}_{25}$ cluster (a), in the core of icosahedral $\mathrm{PdAu}_{12}$ cluster (b), in the core of cuboctahedral $\mathrm{PdAu}_{12}$ cluster (c), on the surface of $\mathrm{Au}_{25}$ cluster (d), on the surface of icosahedral $\mathrm{PdAu}_{12}$ cluster (e), on the surface of cuboctahedral $\mathrm{PdAu} 12$ cluster ( $f$ ) or in the staple motif of $\mathrm{Au}_{25}$ cluster (g), as well as for Pd, attached to a single thiol motif ( $h$ ) or two thiol motifs (i), and for four-coordinated Pd in Pd-S motif as in PdS (j). Spectra a, $d$ and g were used for further analysis and for construction of reference spectra using BSS approach. Obtained corresponding BSS components are shown as $a^{*}, d^{*}$ and $g^{*}$, correspondingly.

numbers significantly.

XANES analysis is a more robust approach, as we show here. In Fig. 6 we show Pd K-edge XANES spectra, calculated for different possible environments of $\mathrm{Pd}$ atom in the investigated samples. Structure models, used for FEFF calculations, in this case were based on the equilibrium structure of thiolated $\mathrm{Au}_{25}$ clusters, as obtained by diffraction analysis of single crystals ${ }^{56}$, but one of the gold atoms was replaced by Pd atom. In Fig. 6 we compare the results of XANES modeling for Pd atom located in the core, at the surface and in the staple motif of $\mathrm{Au}_{25}$ cluster. In addition, we calculate XANES spectra for Pd-doped gold cluster without thiols (i.e., for icosahedral $\mathrm{PdAu}_{12}$ nanocluster), and, again, we compare the XANES spectra for cases, when Pd located in the core 
or at the surface of such cluster. Moreover, we also present the results of XANES calculations for $\mathrm{PdAu}_{12}$ nanocluster with cuboctahedral shape, for an isolated Pd atom, attached to a single thiol motif or two thiol motifs, and for four-coordinated Pd atom in isolated Pd-S motif, similar to that in palladium sulphide.

Note that XANES spectra for Pd, located in the core of $\mathrm{PdAu}_{12}$, models with two different geometries are close to the spectrum of $\mathrm{Pd}$, located in the core of $\mathrm{Au}_{25}$ particle. Hence our analysis will not be able to distinguish between these different Pd environments. Similarly, XANES for Pd, located on the surface of $\mathrm{Au}_{25}$, is close to the XANES spectra for Pd on the surface of $\mathrm{PdAu}_{12}$ clusters. Moreover, XANES for Pd, located in the staple motif of $\mathrm{Au}_{25}$, is close to those for a single Pd atom, attached to a single or several thiol motifs, or incorporated in four-coordinated Pd-S motif.

The observed similarities in XANES spectra for quite different models, on one hand, limit the amount of information that can be extracted from XANES analysis, but on the other hand demonstrate the robustness of this approach. Indeed, even if the arrangement of atoms in the real sample is significantly distorted and/or not coinciding with the positions of atoms in our model (e.g., if the actual size of clusters is larger than the one assumed in XANES modelling), we still can obtain reliable quantitative information. For instance, we will not be able to distinguish between models with two-coordinated and four-coordinated Pd atoms in Pd-S motifs, but the reduction of corresponding contribution to the total XANES can be detected. Hence the total concentration of such motifs in the sample can be analyzed and their partial disappearance upon chemical or thermal treatment can be quantified. Such information cannot be obtained easily from EXAFS analysis of heterogenous material.

For further analysis of experimental data we used only three representative calculated XANES spectra: XANES signal for Pd (i) in the core of $\mathrm{Au}_{25}$ cluster, (ii) at the surface of $\mathrm{Au}_{25}$ cluster and (iii) within the staple motif of $\mathrm{Au}_{25}$ cluster. Note that the differences between these spectra are larger than systematic error of ab-initio simulations for Pd K-edge XANES, as estimated in our calculations for bulk reference materials.

\section{Results and discussion}

In Figs. 7 and 8 we compare the results of LCA fits of experimental data for as prepared and treated Pd-doped $\mathrm{Au}_{25}$ samples, obtained in a reaction of thiolated $\mathrm{Au}_{25}$ with 6 equiv Pd acetate and 0.5 equiv Pd acetate, correspondingly. As previously, we performed fits using two different approaches. In the first case (red dashed lines in Figs. 7 and 8) as standards for linear combination analysis we directly used theoretical XANES spectra, calculated by FEFF code. In the second case (green solid lines) we employed BSS-like procedure, as described above. As input data for BSS procedure in this case we used all 6 available experimental spectra for Pd-doped $\mathrm{Au}_{25}$ samples, since we expect that occupation of different sites, available for Pd atom, will be different in these 6 samples. Three standards for LCA analysis then were obtained as a linear transformation of the six available experimental spectra, requiring the best possible agreement between obtained standards and results of ab-initio XANES simulations for Pd in the

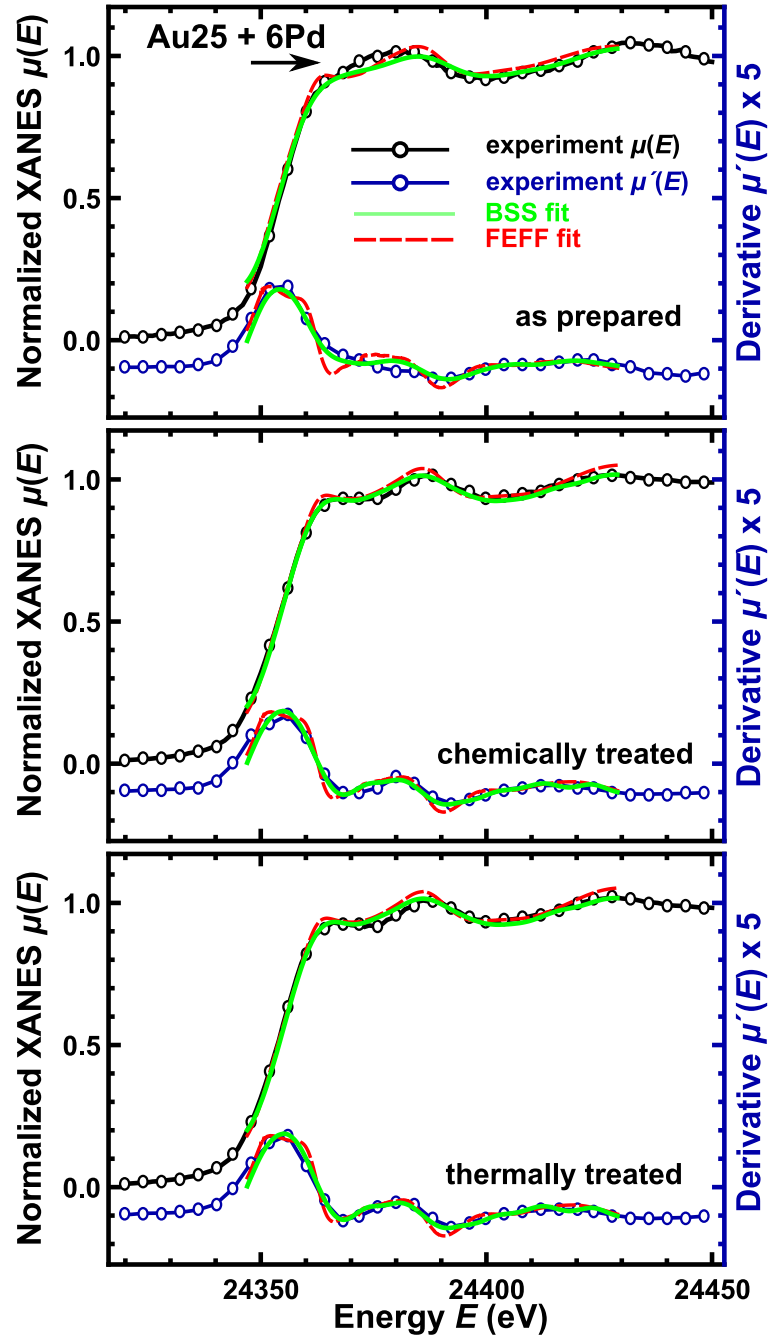

Fig. 7 (Color online) Pd K-edge XANES spectra for samples, obtained in a reaction of thiolated $\mathrm{Au}_{25}$ clusters and 6.0 equiv of $\mathrm{Pd}$ acetate: experimental spectra and their fits using linear combinations of standards that are obtained in FEFF calculations and in BSS-like procedure.

core, at the surface and within the staple motif of $\mathrm{Au}_{25}$ cluster. The obtained standards are shown as green solid lines in Fig. 6 . The results of LCA fits of experimental data using these standards are shown in Figs. 7 and 8.

By construction, the LCA fits using standards, obtained in BSSlike procedure, give better agreement with experimental data than LCA fits that employ as standards the results of XANES calculations directly. The only notable difference between LCA fits with BSS standards and experimental data is visible in the shape of the first broad peak for as-prepared samples (indicated by black arrow in Fig. 7). This may be an indication that additional Pd-containing species or more complex atomic arrangements are present in as-treated samples, as also suggested by EXAFS data.

The weights of different contributions to the total XANES spectrum, as obtained by our analysis using BSS standards, are summarized in Table 2. As expected from EXAFS data, in the asprepared samples the contribution of Pd within staple motif (or within other Pd-S motifs) is dominating. Nevertheless, our re- 


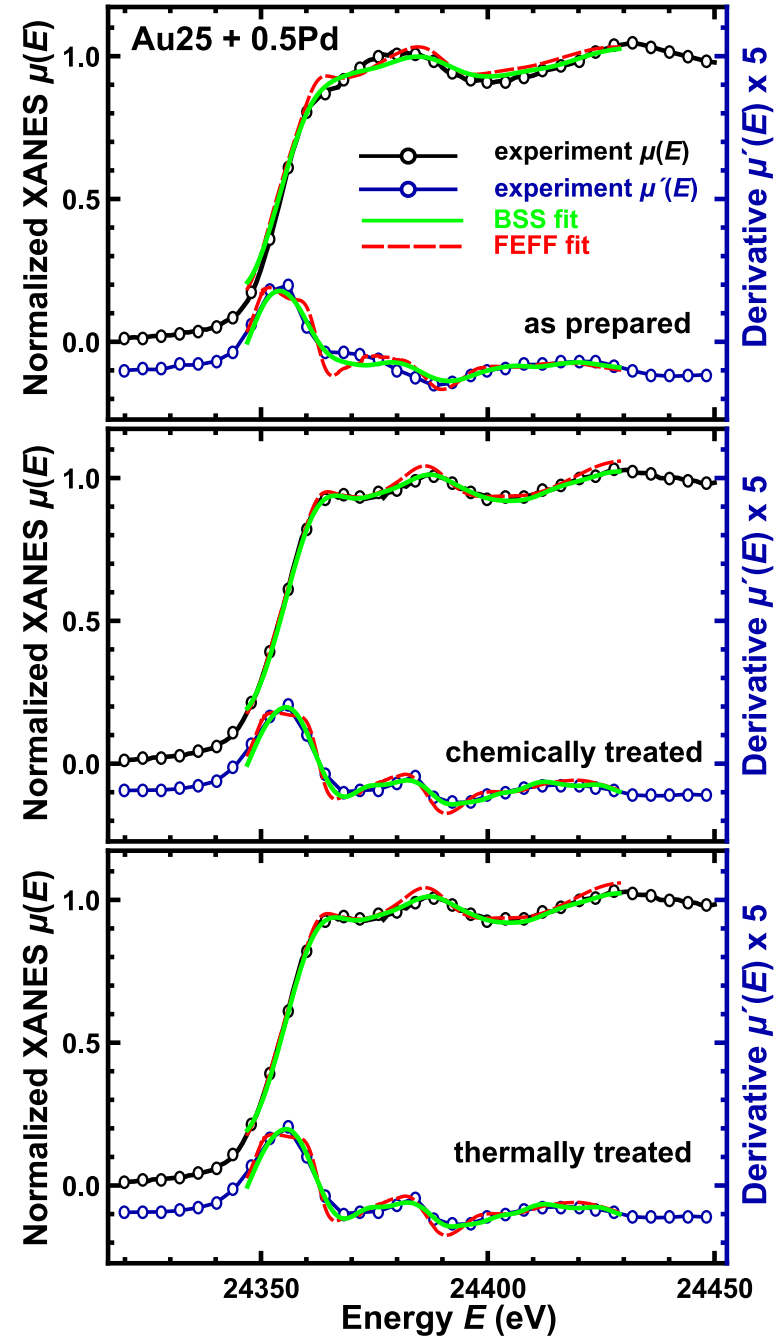

Fig. 8 (Color online) Pd K-edge XANES spectra for samples, obtained in a reaction of thiolated $\mathrm{Au}_{25}$ clusters and 0.5 equiv of $\mathrm{Pd}$ acetate: experimental Pd K-edge XANES and their fits using linear combinations of standards that are obtained in FEFF calculations and in BSS-like procedure.

sults suggest that contribution of Pd, located in the core of cluster, is also present in the as-prepared sample, in agreement with the results of theoretical simulations ${ }^{43}$ and previous studies for samples, where co-reduction synthesis method was employed $46,47,58$. Note also that the ratio of occupancies of staple and core sites is similar for samples, obtained in a reaction of thiolated $\mathrm{Au}_{25}$ clusters with 6 equiv Pd acetate and 0.5 equiv Pd acetate, indicating that the final product of the reaction is not sensitive to the ratio of Pd-containing and Au-containing precursors, as observed for similar system in Ref. ${ }^{46}$.

Both chemical and thermal treatments, as expected, reduce the contribution of Pd within staple motif, hence one may expect that the thiol removal and catalyst activation was partially successful for both treatment methods. A significant Pd-S contribution is, nevertheless, still present in the treated samples. Note also that within error bars of our analysis we do not observe changes in the occupancy of core sites by Pd atoms after chemical and thermal treatment. Our results suggest that the Pd released from staple- motifs is accumulated on the surface of the cluster. This information is important for planning catalytic experiments with such systems because of the enhanced catalytic activity and selectivity of bimetallic compositions to many reactions ${ }^{42,59}$.

Table 2 Pd K-edge XANES fitting results for samples, obtained in a reaction of thiolated $\mathrm{Au}_{25}$ clusters and 6 equiv $\mathrm{Pd}$ acetate ("Au25 + 6 $\mathrm{Pd}$ ") and 0.5 equiv $\mathrm{Pd}$ acetate ("Au25 + 0.5 Pd"). Standards, obtained in BSS-like procedure, are employed.

\begin{tabular}{llll}
\hline & $w_{\text {core }}$ & $w_{\text {surface }}$ & $w_{\text {staple }}$ \\
\hline Au25 + 6 Pd, as prepared & $0.2(1)$ & $0.0(1)$ & $0.8(1)$ \\
Au25 + 6 Pd, chemically treated & $0.2(1)$ & $0.3(1)$ & $0.5(1)$ \\
Au25 + 6 Pd, thermally treated & $0.2(1)$ & $0.4(1)$ & $0.4(1)$ \\
\hline Au25 + 0.5 Pd, as prepared & $0.2(1)$ & $0.0(1)$ & $0.8(1)$ \\
Au25 + 0.5 Pd, chemically treated & $0.3(1)$ & $0.2(1)$ & $0.5(1)$ \\
Au25 + 0.5 Pd, thermally treated & $0.2(1)$ & $0.3(1)$ & $0.5(1)$ \\
\hline
\end{tabular}

\section{Conclusions}

We reported a method of structural refinement of experimental XANES spectra obtained in heterogeneous systems by using theoretical standards. This novel approach can be used not only to determine the ratios of different species in mixtures, when the experimental XANES data for reference materials are not available, but also to determine the occupancies of non-equivalent sites within the investigated structures. We also provided a method to evaluate the accuracy of the proposed speciation approach.

\section{Acknowledgments}

We gratefully acknowledge support from the National Science Foundation under the DMREF program Grant No. CHE1534184. This research used resources of the Center for Functional Nanomaterials, which is a U.S. DOE Office of Science Facility, at Brookhaven National Laboratory under Contract No. DESC0012704. Synchrotron measurements described in this work were performed at the Canadian Light Source, which is supported by the Natural Sciences and Engineering Research Council of Canada, the National Research Council Canada, the Canadian Institutes of Health Research, the Province of Saskatchewan, Western Economic Diversification Canada, and the University of Saskatchewan.

\section{References}

1 D. E. Sayers, E. A. Stern and F. W. Lytle, Phys. Rev. Lett., 1971, 27, 1204.

2 P. A. Lee, P. H. Citrin, P. T. Eisenberger and B. M. Kincaid, Rev. Mod. Phys., 1981, 53, 769.

3 J. J. Rehr and R. Albers, Rev. Mod. Phys., 2000, 72, 621.

4 J.-D. Grunwaldt and A. I. Frenkel, Synchrotron Radiat. News, 2009, 22, 2-4.

5 A. I. Frenkel and J. A. van Bokhoven, J. Synchrotron Radiat., 2014, 21, 1084-1089.

6 A. O. Er, J. Chen and P. M. Rentzepis, J. Appl. Phys., 2012, 112, 031101.

7 A. I. Frenkel, J. A. Rodriguez and J. G. Chen, ACS Catal., 2012, 2, 2269-2280.

8 J. Itié, A. Polian, D. Martinez, V. Briois, A. Di Cicco, A. Filip- 
poni and A. San Miguel, Le Journal de Physique IV, 1997, 7, C2-31.

9 A. Di Cicco, F. D’Amico, G. Zgrablic, E. Principi, R. Gunnella, F. Bencivenga, C. Svetina, C. Masciovecchio, F. Parmigiani and A. Filipponi, J. Non-Cryst. Solids, 2011, 357, 2641-2647.

10 Y. Ping, F. Coppari, D. Hicks, B. Yaakobi, D. Fratanduono, S. Hamel, J. Eggert, J. Rygg, R. Smith, D. Swift et al., Phys. Rev. Lett., 2013, 111, 065501.

11 A. I. Frenkel, A. Yevick, C. Cooper and R. Vasic, Annu. Rev. Anal. Chem., 2011, 4, 23-39.

12 B. S. Clausen and J. K. Nørskov, Top. Catal., 2000, 10, 221230.

13 A. Witkowska, A. Di Cicco and E. Principi, Phys. Rev. B, 2007, 76, 104110.

14 A. Yevick and A. I. Frenkel, Phys. Rev. B, 2010, 81, 115451.

15 S. T. Chill, R. M. Anderson, D. F. Yancey, A. I. Frenkel, R. M. Crooks and G. Henkelman, ACS Nano, 2015, 9, 4036-4042.

16 A. I. Frenkel, Phys. Scripta, 2015, 90, 098004.

17 A. Ankudinov, J. Rehr, J. J. Low and S. R. Bare, J. Chem. Phys., 2002, 116, 1911-1919.

18 J. Rehr and A. Ankudinov, Coordin. Chem. Rev., 2005, 249, 131-140.

19 G. Ciatto, A. Di Trolio, E. Fonda, P. Alippi, A. Testa and A. A. Bonapasta, Phys. Rev. Lett., 2011, 107, 127206.

20 Q. Ma, J. Prater, C. Sudakar, R. Rosenberg and J. Narayan, J. Phys.: Condens. Matter, 2012, 24, 306002.

21 A. Kuzmin and J. Chaboy, IUCrJ, 2014, 1, 571-589.

22 A. Ankudinov, B. Ravel, J. Rehr and S. Conradson, Phys. Rev. B, 1998, 58, 7565 .

23 D. Bazin and J. Rehr, J. Phys. Chem. B, 2003, 107, 1239812402.

24 J. J. Kas, K. Jorissen and J. J. Rehr, X-Ray Absorption and X-Ray Emission Spectroscopy: Theory and Applications, John Wiley \& Sons, 2016, p. 51.

25 S. Della Longa, A. Arcovito, M. Girasole, J. Hazemann and M. Benfatto, Phys. Rev. Lett., 2001, 87, 155501.

26 M. Benfatto, A. Congiu-Castellano, A. Daniele and S. Della Longa, J. Synchrotron Radiat., 2001, 8, 267-269.

27 G. Smolentsev, A. Soldatov and M. Feiters, Phys. Rev. B, 2007, 75, 144106.

28 G. Smolentsev, S. Canton, J. Lockard, V. Sundstrom and L. Chen, J. Electron Spectrosc., 2011, 184, 125-128.

29 M. Benfatto and C. Meneghini, Synchrotron Radiation, Springer, Berlin, Heidelberg, 2015, pp. 213-240.

30 S. Wasserman, Le Journal de Physique IV, 1997, 7, C2-203.

31 A. I. Frenkel, O. Kleifeld, S. R. Wasserman and I. Sagi, J. Chem. Phys., 2002, 116, 9449-9456.

32 X. Wang, J. C. Hanson, A. I. Frenkel, J.-Y. Kim and J. A. Rodriguez, J. Phys. Chem. B, 2004, 108, 13667-13673.

33 A. Manceau, M. Marcus and T. Lenoir, J. Synchrotron Radiat., 2014, 21, 1140-1147.

34 C. Ruckebusch and L. Blanchet, Anal. Chim. Acta, 2013, 765,
35 W. H. Cassinelli, L. Martins, A. R. Passos, S. H. Pulcinelli, C. V. Santilli, A. Rochet and V. Briois, Catal. Today, 2014, 229, 114 122.

36 A. Voronov, A. Urakawa, W. van Beek, N. E. Tsakoumis, H. Emerich and M. Rønning, Anal. Chim. Acta, 2014, 840, 20-27.

37 H. W. Carvalho, S. H. Pulcinelli, C. V. Santilli, F. Leroux, F. Meneau and V. Briois, Chem. Mater., 2013, 25, 2855-2867.

38 D. D. Lee and H. S. Seung, Nature, 1999, 401, 788-791.

39 A. Hyvärinen and E. Oja, Neural networks, 2000, 13, 411-430.

40 S. Wang, X. Meng, A. Das, T. Li, Y. Song, T. Cao, X. Zhu, M. Zhu and R. Jin, Angew. Chem., 2014, 53, 2376-2380.

41 S. Xie, H. Tsunoyama, W. Kurashige, Y. Negishi and T. Tsukuda, ACS Catal., 2012, 2, 1519-1523.

42 H. Qian, D.-e. Jiang, G. Li, C. Gayathri, A. Das, R. R. Gil and R. Jin, J. Am. Chem. Soc., 2012, 134, 16159-16162.

43 K. A. Kacprzak, L. Lehtovaara, J. Akola, O. Lopez-Acevedo and H. Hakkinen, Phys. Chem. Chem. Phys., 2009, 11, 7123-7129.

44 A. Shivhare, K. E. Lee, Y. Hu and R. W. Scott, J. Phys. Chem. C, 2015, 119, 23279-23284.

45 A. Shivhare, S. J. Ambrose, H. Zhang, R. W. Purves and R. W. Scott, Chem. Commun., 2013, 49, 276-278.

46 C. A. Fields-Zinna, M. C. Crowe, A. Dass, J. E. Weaver and R. W. Murray, Langmuir, 2009, 25, 7704-7710.

47 Y. Negishi, W. Kurashige, Y. Kobayashi, S. Yamazoe, N. Kojima, M. Seto and T. Tsukuda, J. Phys. Chem. Lett., 2013, 4, 3579-3583.

48 B. Ravel and M. Newville, J. Synchrotron Radiat., 2005, 12, 537-541.

49 J. J. Rehr, J. J. Kas, F. D. Vila, M. P. Prange and K. Jorissen, Phys. Chem. Chem. Phys., 2010, 12, 5503-5513.

50 K.-T. Park, D. Novikov, V. Gubanov and A. Freeman, Phys. Rev. B, 1994, 49, 4425.

51 N. E. Brese, P. J. Squattrito and J. A. Ibers, Acta Crystallogr. C, 1985, 41, 1829-1830.

52 A. Cichocki, R. Zdunek and S.-i. Amari, Acoustics, Speech and Signal Processing, 2006. ICASSP 2006 Proceedings. 2006 IEEE International Conference on, 2006, pp. V-V.

53 J. Felten, H. Hall, J. Jaumot, R. Tauler, A. de Juan and A. Gorzsás, Nat. Protoc., 2015, 10, 217-240.

54 J. A. Nelder and R. Mead, Comput. J., 1965, 7, 308-313.

55 M. A. Tofanelli, T. W. Ni, B. D. Phillips and C. J. Ackerson, Inorg. Chem., 2016, 55, 999-1001.

56 M. W. Heaven, A. Dass, P. S. White, K. M. Holt and R. W. Murray, J. Am. Chem. Soc., 2008, 130, 3754-3755.

57 M. Dasog, W. Hou and R. W. Scott, Chem. Commun., 2011, 47, 8569-8571.

58 S. L. Christensen, M. A. MacDonald, A. Chatt, P. Zhang, H. Qian and R. Jin, J. Phys. Chem. C, 2012, 116, 2693226937.

59 M. Sankar, N. Dimitratos, P. J. Miedziak, P. P. Wells, C. J. Kiely and G. J. Hutchings, Chem. Soc. Rev., 2012, 41, 8099-8139. 